\title{
Glycoconjugates and Glycomimetics as Microbial Antiadhesives
}

\author{
Sara Sattin and Anna Bernardi* \\ Università degli Studi di Milano, Dipartimento di Chimica, via Golgi 19, 20133 Milano, Italy \\ *e-mail: anna.bernardi@unimi.it
}

\begin{abstract}
Microbial adhesion is an essential step of infections and is mediated primarily by proteincarbohydrate interactions. Antagonists of such interactions have become a promising target for antiadhesive therapy in a number of infective diseases. Monovalent protein-sugar interactions are often weak, and most successful antiadhesive materials consist of multivalent glycoconjugates. Although often very effective in hampering microbial adhesion, natural epitopes often show limited resistance to enzymatic degradation. The use of carbohydrate mimics (glycomimetics) as a replacement for natural sugars potentially allows to achieve higher metabolic stability and also higher selectivity towards the desired protein target. In this review we will describe the state of the art on the design and synthesis of glycoconjugates and glycomimetics employed for the construction of antiadhesive biomaterials.
\end{abstract}

\section{Keywords}

Adhesins, lectins, FimH, DC-SIGN, antiadhesion therapy 


\section{Microbial adhesion and carbohydrates}

Bacterial adhesion to host cells is essential for microbial pathogenesis and is initiated by molecular recognition events at the host-guest interface. Efficient adhesion allows the pathogen to escape the natural cleansing mechanisms of the host and provides a means for access to nutrients, delivery of toxins and ultimately colonization and invasion of the host, including biofilm formation [1]. In most cases, molecular recognition is triggered by specific carbohydrate-protein interactions involving glycoconjugates and sugar-binding protein (lectins). The lectin involved can be displayed either on the bacteria or on the host surface. Bacterial adhesins (see Glossary), located on the bacterial surface or on pili and fimbriae, interact with specific glycans on host tissues to initiate colonization. Bacteria also use adhesins to adhere to other microbial cells as a prerequisite for biofilm formation. Conversely, specific host lectins, particularly in the immune system, recognize pathogens by interaction with their surface glycans. This step, designed for productive pathogen processing and clearance, is often exploited by the pathogen to colonize the host. Thus, it has been proposed that appropriate neo-glycoconjugates able to interfere with carbohydrate-protein recognition could be used to inhibit microbial adhesion in the very early stages of an infection. This approach strives to prevent colonization and possibly even to reverse biofilm formation, but it does not aim to kill the invading pathogen, so it should not exert selective pressure leading to resistance [2]. As bacterial resistance to antibiotic treatment is increasing and becoming a pressing public-health concern, anti-adhesive therapies are emerging as a valuable alternative or complementary approach.

Individual interactions between proteins and sugars are usually very weak, a flaw often overcome in living systems by multivalency on both the glycan and the lectin side, so that high avidity is reached. This effect, often referred to as "the glycan cluster effect" or "the velcro effect" largely depends on the specific features of lectin binding sites, which tend to be flat, large and 
exposed to the solvent. Effective antiadhesive therapy requires either high affinity monovalent lectin ligands capable of outperforming glycan clusters, or multivalent structures incorporating several copies of ligands of moderate affinity on a polyvalent scaffold (dendrimer, polymer, nanoparticle). Depending on the nature of the lectin involved, a large number of glycomimetic and neo-glycoconjugate structures have been proposed as antagonists of sugar-protein interaction events initiating bacterial and viral infections and recent comprehensive reviews are available [36]. Among the many reported examples, in this paper we will focus on two applications of the antiadhesive approach targeting the recognition of host glycans by a bacterial adhesin or the recognition of microbial glycans by a human lectin. These two examples, that have been thoroughly investigates in recent years, illustrate how an antiadhesive approach can be used against different types of pathogens exploiting the same fundamental (bio)chemical mechanism and targeting either host or microbial sugar receptors. In the first case, the development of inhibitors of adherent-invasive (AIEC) and uropathogenic (UPEC) E. coli represents an alternative and/or a complementary antibacterial strategy against widespread and difficult-to-treat infections,

which are increasingly becoming resistant to classical antibiotic therapies. With the second paradigmatic example we aim to illustrate the progress made towards prophylactic antiviral agents targeting DC-SIGN (dendritic cell-specific ICAM-3 grabbing non-integrin), a receptor of the human immune system that, although discovered only 15 years ago, is rapidly becoming and established target for microbial infections.

\section{Inhibitors of adherent-invasive and uropathogenic $E$. coli}

Infections of the urinary tract are most frequently caused by UPEC adhering to $\alpha$ mannopyranosyl ligands on the surface of host's urothelial cells. The adhesin involved is called FimH and is located on the tip of bacterial type-1 fimbriae, rod-like fibers $1-2 \mu \mathrm{m}$ long and $7 \mathrm{~nm}$ 
thick, that protrude from the bacterium body. FimH contains a mannose-specific lectin domain, which adheres to terminal mannosides of two glycoproteins, uroplakin and $\alpha_{3} \beta_{1}$ integrin, abundantly expressed by urothelial cells. FimH also appears to control adhesion of adherentinvasive $E$. coli (AIEC) isolated from Crohn's disease patients.

It was established early on that mannosides bearing aromatic aglycones can be used as antagonists of FimH mediated bacterial adhesion [7]. Crystallographic studies [8] (pdb: 1UWF, 1TR7) showed that the lectin domain of the adhesin contains a relatively deep sugar-binding pocket lined by aromatic lipophylic residues at the rim (the tyrosine gate, formed by Tyr48, Tyr137 and Ile52) and established butyl $\alpha$-D-mannoside 1 (Figure 1a) as a strong antagonist. Optimization of the aglycone chain length led to the discovery of $n$-heptyl $\alpha$-D-mannoside ( $\alpha$-D-HM, 2, Figure 1a) as a nanomolar FimH antagonist [9]. Recent structural studies [10] have called into question the importance of the tyrosine gate in the recognition process of mannosides by the full FimH protein. Indeed, NMR relaxation analysis and small-angle scattering X-ray studies revealed that $\alpha-D-H M 2$ promotes dimerization of FimH CRD, a process that may lead to overestimation of its binding affinity in in vitro studies. Additionally, a Y48A mutant of FimH was found to possess binding properties very similar to the wild type protein. However, the selectivity of aryl mannosides for FimH and against a panel of human mannose-binding lectins, a key element for in vivo applications, was shown to be determined by the structure of the aglycone $[11,12]$.

Dissociation constants in the $\mathrm{nM}$ range have been routinely achieved with both polyvalent and monovalent compounds, and, depending on the application, both monovalent mannosides and mannosylated high-valency materials are being developed as FImH antagonists. Prevention and treatment of urinary tract infection by UPEC requires orally active FimH antagonists, absorbed in the intestine and renally excreted, with finely tuned pharmacokinetic properties, so as to achieve an optimal balance between affinity and duration of the therapeutic effect in the bladder. 
This is typically pursued with monovalent aryl mannosides [13-17] targeted to the FimH binding site by their mannose component and carrying extended aromatic aglycones that establish high affinity interaction with the tyrosine gate (3-5, Figure 1a). Extensive medicinal chemistry programs are under way, including the use of prodrugs and bioisosters to achieve optimal pharmacological profile of these structures [18]. These studies recapitulate the challenges faced by development of "traditional" drugs starting from carbohydrates structures, with specific problems and difficulties that are not encountered in other medicinal chemistry areas.

A large number of multivalent structures targeting FimH have been reported for applications where intestinal absorption is not required (e.g. against gut-colonizing AIEC), or when FimH adhesion is exploited to achieve bacterial aggregation and biofilm disruption. They vary from medium-sized scaffolds [19-23], to dendrimers [3, 24], polymers [25] and nanoparticles [2629]. Despite the spacing between fimbriae can vary between strains and depends on growth conditions [30], only the largest of these materials are likely to engage more than one protein at the time, because the FimH binding site can interact with only one mannose residue at the time and each of the fimbriae carries only a single copy of FimH. In most other systems, the multivalency effects observed are not likely to depend on chelation, but rather on increased local concentration of the ligand (statistical rebinding). Affinity improvements have been measured also for materials of moderate valency (up to 12 mannose units) in inhibition experiments with isolated FimH

E. coli aggregation in water solution, using large polyvmannosylated constructs is under active examination for detection and removal from polluted water [27] and as a model system for anti-infective studies in vivo [21] or ex vivo $[25,29]$. Glyconanodiamonds (GNDs) have been used towards this end. The surface of nanodiamonds (NDs) [31] 7 (ca. $5 \mathrm{~nm}$ in diameter) was functionalized by a Diels-Alder reaction with a ortho-quinodimethide generated in situ from 1,2- 
dibromomethylbenzene (Figure $1 \mathrm{~b}$ ). Sulfonation and partial reduction of the sulfonic acid groups yielded nanoparticles that are stable as colloids in water solution (due to the charges of the residual sulfonic acid moieties) and can be linked to allyl mannoside $\mathbf{1 0}$ by thiol-ene reaction [34]. Alternatively, the trivalent mannoside cluster 13 was grafted to diamonds carrying carboxy groups by amide synthesis with the amino group at its focal point (Figure 1c). The glycosylated NDs were shown to perform as mimics of host cells surface, and mannosylated samples were found to specifically adhere to fimbriated bacteria, agglutinating them from polluted water samples. Some aspecific effects of the diamond were also observed. However, only the mannosylated particles gave rise to stable aggregates that could not be dispersed by shaking and could be filtered away with commercial filters with pore size $10 \mu \mathrm{M}$ (too large to retain bacteria themselves). This type of materials can be regenerated by concentrated mannose solutions and therefore should be appropriate to build continuous filtration cartridges. The NDs 14, that carry the trivalent mannose cluster, displayed higher activity in the aggregation assay, likely as an effect of the increased local concentration of the ligands, which should reduce the off rate of the protein from the surface. Nonetheless, NDs 11, which are synthetically more accessible, were used for most of the reported applications [27].

\section{Figure 1}

The use of GNDs as potent and specific anti-adhesives against $E$. coli adhesion to human bladder cells and their interference with biofilm formation was recently reported [29]. Functionalization of surface hydroxyl groups of commercial NDs followed by sugar grafting yielded particles that were coated either with mannose itself or with a trivalent cluster of a mannose mimic ( $\alpha$-thiomannoside) (Figure 1e). The latter construct, besides increasing the local density of mannose residues, is more stable against glycosidase-mediated hydrolysis. Both types of particles did not display toxicity against eukaryotic cells and showed marked anti-adhesive activity in cellbased assays. In addition, they were also able to inhibit E. coli induced biofilm formation on 
polyvinylchloride (PVC) surfaces in a concentration dependent and time-dependent manner. Strikingly, the trimannoside cluster $\mathbf{2 0}$ was also very active in this assay (only a factor of 2 less active than the full ND construct, 21), an unexpected finding because low-valency mannosides have rarely been reported to disrupt $E$. coli biofilms.

While this paper was under review, an E. coli capture device built on graphene sheets was reported [80]. The device self-assembles treating adamantyl-functionalized graphene with heptamannosylated $\beta$-cyclodextrin (ManCD) and it captures $E$. coli wrapping it up in the graphene sheet in a reversible fashion. Interestingly, the thermal IR-absorption properties of graphene allows to exploit IR-laser irradiation to kill the captured bacteria, thus providing a new strategy towards disinfection.

AIEC eradication from the intestinal tract also does not require adsorption and monovalent mannosides have been reported as effective agents in this application [32]. Recently [25], polymers of different size and topology were synthesized by co-polymeraztion of $\mathrm{N}-(\alpha-$ mannopyranosyloxy)heptyl]methacrylamide (HMM) with different monomers, to obtain a set of defined linear or star glycopolymers carrying from 31 to 269 copies of $\alpha$-D-HM 2. Interaction of the polymers with FimH adhesin was studied by DLS and AFM, revealing the formation of large (1400 nm length $\times 210 \mathrm{~nm}$ diameter) cylindrical aggregates, showing the ability of each glycopolymer to accommodate multiple copies of the adhesin. Consistently, the polymers were powerful agglutinators of piliated $E$. coli, yielding clusters whose size increases with the concentration of the glycopolymer chains. The antiadhesive properties of the polymers were tested both in pre- and post-incubation assays of AIEC to T84 intestinal epithelial cells, as a model for AIEC adhesion to intestinal host cells in Crohn's disease patients. Compared to the monovalent counterparts, the polymers produced a significant decrease of residual bacterial adhesion that was optimal for linear glycopolymers over the star-shaped ones and improved with the length of the 
polymer (see Figure 1f for the most active linear glycopolymer L188, 22). Control experiments with similar polymers carrying ethyl mannoside rather than $\mathbf{2}$ showed no activity and confirmed the requirement for optimization of the individual ligands and/or of their spacing from the bulk of the polymer chain. The best polymer $\mathbf{2 2}$ was tested in an ex vivo experiment and was shown to prevent AIEC adhesion to a model of Crohn's disease colonic loop (61\% decrease of bacteria adhering to colonic mucosa). One of the most interesting conclusions of this study is that the antiadhesive and cell aggregation properties of the polymers did not correlate with dissociation constants measured in classical interaction studies between FimH and the glycopolymers. Indeed, ELISA assays measuring inhibition of FimH binding to a highly mannosylated protein (RNAseB) showed the highest level of inhibition for monovalent $\alpha$-D-HM 2 and no gain of affinity was observed with the polymeric ligands. Whether this depends on the pathogen scavenging ability of the polymers or on the protein dimerization ability of $\mathbf{2}$ [10], this result suggests that many factors beyond ligand affinity need to be taken into account when designing anti-adhesive therapeutic strategies.

\section{Prophylactic antiviral agents targeting DC-SIGN.}

DC-SIGN (dendritic cell-specific ICAM-3 grabbing non-integrin), or CD209, is a tetrameric transmembrane protein expressed by immature dendritic cells as a pathogen recognition receptor (PRR). It belongs to the family of calcium dependent (C-type) lectins and binds to pathogens by specifically recognizing highly-glycosylated structures displayed at their surface [36-39]. PRR are used by dendritic cells to internalize invading pathogens and degrade them in lysosomal compartments. The resulting fragments are exposed on their surface bound to the Major Histocompatibility Complex (MHC) and presented to CD4+ T lymphocytes to initiate the specific immune response. In a seminal paper [36], it was shown that HIV takes advantage of DC-SIGN to invade the host immune system: after the initial interaction, HIV escapes the normal degradation 
pathway so that whole virus particles are transmitted to CD4+ T cells, a process called infection in trans. The detailed molecular mechanisms by which this occurs have not been fully elucidated, however it has also been repeatedly shown that blocking HIV recognition by DC-SIGN effectively abrogates in trans infection. Other pathogens have been shown to similarly hijack DC-SIGN, among them the Ebola and Dengue viruses [39].

The natural ligand of DC-SIGN on HIV surface is the high mannose glycan, (Man $)_{9}(\mathrm{GlcNAC})_{2}$ $\left(\mathrm{Man}_{9}\right.$, Figure 2), a branched glycan from the gp120 envelope protein. The protein also recognizes fucose residues in oligosaccharides of the Lewis antigen type (Figure 2). The glycans interact primarily with a $\mathrm{Ca}^{2+}$ ion exposed at the surface of DC-SIGN carbohydrate recognition domain (CRD). X-ray data are available for complexes of DC-SIGN CRD with mannose oligosaccharides, and with the fucosylated oligosaccharide Lewis ${ }^{\mathrm{x}}$ [40-43].

\section{Figure 2}

Dermal dendritic cells in vaginal, cervical and rectal mucosae express DC-SIGN and are among the first cells encountered by HIV. Hence, a number of groups are working to design DCSIGN antagonists [44-50] as topical "microbicides" (or, more appropriately, 'virustats' [51]) blocking viral adhesion and entry in sexually transmitted HIV infection. To this end, two additional issues need to be taken into account. First, the antagonists ought to be selective for DC-SIGN and against the other numerous mannose specific human lectins operating in the immune system. In particular, attention has been drawn to selectivity against Langerin, a mannose specific C-lectin found in Langerhans cells that captures HIV and mediates its internalization into Birbeck granules, which results in rapid degradation of the virus. Arguably, a successful topical inhibitor of HIV infection should avoid Langerin and efficiently block recognition by DC-SIGN on the underlying mucosal DCs [52]. A more subtle issue is represented by the dual function of DC-SIGN, which appears to mediate both tolerance and immunity, depending on the context of the initial 
interaction $[53,54]$. Thus, the downstream effects of engaging DC-SIGN with CRD-targeted ligands are still not fully predictable and should be carefully analyzed.

Multivalency is a key feature of DC-SIGN HIV interaction, since the DC-SIGN tetramers presents 4 CRDs to the highly glycosylated gp120 protein. Although small molecules have also been reported as efficient DC-SIGN antagonists [55], the vast majority of the reported antiviral agents are based on a multivalent presentation of mannose-based or fucose-based carbohydrates or carbohydrate mimics on appropriate multimeric scaffolds.

\section{Mannose-based materials}

Proof of concept was initially achieved using mannose monosaccharides as the active ligand on high-valency constructs, able to compensate for the low affinity of mannose itself. The first polyvalent DC-SIGN antagonists, reported a few years ago, consisted of polydisperse Boltorn type dendritic polyester platforms functionalized with 16 or 32 copies of mannose. The inhibitory activity of these compounds was initially studied in a pseudo-typed Ebola viral particle infection model. The third generation dendrimer (32 mannose units) was able to inhibit the direct (in cis) and trans infection process in a dose-dependent manner $\left(\mathrm{IC}_{50} 337 \mathrm{nM}\right)$, by blocking the interaction of DC-SIGN with the envelope viral glycoprotein of Ebola, GP1 [56]. For comparison, an $\mathrm{IC}_{50}$ 1.3 $\mathrm{mM}$ was measured for the monovalent ligand $\alpha$-methyl-D-mannopyranoside in the same experiment. The dendrimer was also found to inhibit the interaction between DC-SIGN and gp120 immobilized on an SPR sensor with an $\mathrm{IC}_{50}$ in the micromolar range $\left(\mathrm{IC}_{50} 50 \mu \mathrm{M}\right)$ [57]. To the same end, also water-soluble manno-fullerenes [58], and virus-like manno-dendronanoparticles capable of mimicking pathogens both in size and in their highly glycosylated surfaces [59], were explored (Figure 3a). The latter were built using as platform $Q \beta$, a recombinant capsid protein derived from the bacteriophage $Q \beta$ that spontaneously self-assemble to give icosahedral virus-like particles of 
$25 \mathrm{~nm}$ ca. in diameter [60]. The particle size is ideal to mimic the interaction of viruses with dendritic cells, achieving at the same time a high control over the geometry of the polyvalent carbohydrates display. With a "tag-and-modify" strategy [61] a non-natural aminoacid (Lhomopropargylglycine, $\mathrm{Hpg}$ ) was introduced as a "tag" at position 16 of the Q $\beta$ protein monomer, ideally pointing outwards the assembled particle $\left(\mathrm{Q} \beta-\mathrm{Hpg} 16_{180}\right)$. Modification of the same position by conjugation of tri- or nonavalent manno-dendrons (23 and $\mathbf{2 4}$ respectively, Figure 3a) bearing an azido functionality at the focal point, allowed the controlled display at a very high valency of the mannosylated dendrons. The final constructs $\mathbf{2 5}$ and $\mathbf{2 6}$ bear up to 1,620 units of mannose and were found to be active in low $\mathrm{nM}$ (25) or high pM (26) concentration in the Ebola model infection test.

\section{Figure 3}

Mannosylated glycopolymers of moderate size were also prepared [49,62] and were shown to inhibit DC-SIGN binding to immobilized gp120 (SPR) in the nanomolar range. Dynamic micelles of manno-glycolipids (27, Figure 3b) have been recently reported [63] to inhibit HIV-1 trans-infection mediated by human DCs with $\mathrm{IC}_{50}$ in the low micromolar range and more efficiently than corresponding covalent, polymeric constructs. The authors suggest that the high ligand density and the dynamic properties of the micelles may allow the structure to adjust upon contacting the lectin CRD, in order to establish optimal interactions.

Displaying more complex oligomannosides $\left(\mathrm{Man}_{9}\right.$ or fragments thereof), which have a higher intrinsic affinity than mannose itself for DC-SIGN, proved worthwhile to a certain extent, but at the cost of increased synthetic complexity of the ligand. Glycodendrons displaying 9 copies of $\mathrm{Man}_{4}$ and $\mathrm{Man}_{9}$ instead of mannose led inhibitory activity against gp120 binding to DC-SIGN in the low $\mu M$ range (ELISA assay) [64]. Oligomannoside-functionalized gold nanoparticles were 
tested in DC-SIGN-mediated trans infection of $\mathrm{T}$ cells and showed activity at nanomolar concentration [65]. Interestingly, the presentation of simple linear di-, tri-, and tetraoligosaccharides on the particles afforded results similar to those observed for complex branched penta- and heptamannosides, suggesting that the increased complexity of the active ligand is not required for efficient blockade of the infection.

Efforts toward ligand optimization have been more rewarding when aimed to replacing natural sugars with monovalent glycomimetic structures and/or to designing the multivalent constructs for optimal presentation to the DC-SIGN CRD tetramer. In general, glycomimetics can be designed to be higher affinity ligands than mannose or fucose themselves, without excessively increasing the synthetic complexity. Additionally, they should be more stable against the action of glycosidases, therefore providing longer half-life of the antiadhesive materials in vivo. To this end, a modified shikimic acid derivative $\mathbf{2 8}$ was used as a mannose mimic (Figure 4) supported on a polymer carefully designed to feature the appropriate size (degree of polymerization 29) and ligand density (seven glycomimetics per polymer, 29, Figure 4) to engage multiple copies of DCSIGN [66]. With this design, a low micromolar inhibitor of DC-SIGN binding to mannosylated BSA was obtained, corresponding to a 1000 -fold increase in potency relative to the monomeric inhibitor.

\section{Figure 4}

Another class of mannose-based glycomimetic antagonists are 30 and 31, mimics of 1,2mannobioside and of the linear Man- $\alpha-1,2-$ Man- $\alpha-1,6-$ Man trisaccharide, respectively. They both contain a terminal mannose residue connected to a conformationally locked cyclohexanediol [67] that mimics a mannose unit $-\alpha-1,2$-substituted. Both $\mathbf{3 0}$ and $\mathbf{3 1}$ bind to the $\mathrm{Ca}^{++}$ion in DC-SIGN binding site using the non-reducing end residue (X-ray: $[68,69]$ ), inhibit DC-SIGN binding to 
mannosylated BSA [70], display a measurable selectivity [68, 71] against Langerin, are not cyototoxic and are active in the Ebola infection model. The pseudo-trisaccharide $\mathbf{3 1}$ was the first of these antagonists to be formulated in polyvalent presentations: the tetravalent-Boltorn-type dendron 32 in low micromolar concentrations was shown to block DC-SIGN mediated HIV infection both in cellular and cervical explant models $[70,71]$. Infection of cervical explants by different HIV-1 R5-topic strains was strongly reduced by $\mathbf{3 2}$ and, at higher doses, X-4 topic virus was also blocked. The dendron was also found to induce immune activation and proinflammatory responses, which contribute to its antiviral activity.

\section{Figure 5}

Ligand optimization led to a group of bis-benzylamido derivatives of $\mathbf{3 0}$ that approach the activity of $\mathbf{3 1}$ and display similar or better selectivity against Langerin [72], at a fraction of the synthetic cost. Compound $\mathbf{3 3}$ (Figure 5) was selected among them for the synthesis of dendrimers based on an erythritol core, that were tested for DC-SIGN binding (SPR inhibition assay) and compared to the corresponding versions bearing mannose or 30 [73]. Dendrimers up to the valency of 18 were synthesized; representative structures are shown in Figure 5 (34-36). This represents one of the few reported cases where the activity of similar materials decorated with lectin ligands of different potencies has been studied in a systematic fashion. The results show that even small affinity differences at the monovalent level are amplified by multivalent presentation, as clearly shown by the $\mathrm{IC}_{50}$ values of similar dendrimers of equal valency carrying different monovalent ligands. While $\mathrm{IC}_{50}$ in the low micromolar range are achieved already for hexavalent constructs using the most powerful ligand 33, the other mannosylated and pseudomannosylated dendrimers are significanty less effective, even at higher valency. The hexavalent 
presentation of $\mathbf{3 3}$ in dendrimer 36 provided $100 \%$ inhibition of trans HIV infection at $10 \mu \mathrm{M}$ concentration in a cellular model [73] and stimulated early immune response from DCs [74]. Additionally, 36 also blocked DC-SIGN mediated uptake of Dengue virus (DV) by Raji cells with a low micromolar $\mathrm{IC}_{50}$. [74]

Molecular modelling studies showed that $\mathbf{3 6}$ is actually too small to bridge two adjacent binding sites on the DC-SIGN tetramer, which are separated by about $4 \mathrm{~nm}$. Inclusion of a rigid spacer of appropriate length at the dendrimer core resulted in a significant improvement of activity, presumably by exploiting chelation mechanisms. The rod-like structure $\mathbf{3 7}$ with an $\mathrm{IC}_{50}$ in the nanomolar range (Figure 5) was found to be 40 times more active than $\mathbf{3 6}$ in HIV transinfection studies [75] and has a very interesting potential for actual applications as antiadhesive antiviral agent for topical use.

\section{Fucose-based materials}

As opposed to DC-SIGN, Langerin does not bind fucosylated oligosaccharides. Thus fucosebased materials were pursued mostly to achieve selective DC-SIGN antagonists. Complex fucosylated Lewis-type antigens supported on PAMAM dendrimers (average of 14-16 copies) [52] were analyzed for specificity against Langerin and were indeed found to bind DC-SIGN selectively. Glycodendrimers with larger molecular diameter performed better in solid-phase based and cellular-binding assays and were more efficient in inhibiting gp120 binding to DC-SIGN, despite carrying a similar amount of active ligand. Glycan spacing may be a logical explanation for these findings, but alternative rationales include differences in dendrimer charge, or shape. Interestingly, no signs of activation or maturation on DCs exposed to these glycodendrimers were observed, suggesting that their immunogenicity may be minimal. 


\section{Figure 6}

Extensive work on the design of fucose-based glycomimetics has been reported [76]; these molecules are characterized by the presence of an unnatural $\alpha$-fucosylamide anchor and bind DCSIGN with an affinity similar to that of the Lewis ${ }^{x}$ oligosaccharide. The simplest of these mimics, an $\alpha$-fucosyl- $\beta$-alanyl amide, loaded on a gold nanoparticle platform, gave rise to a potent DC-SIGN targeting device (38, Figure 6) that did not induce dendritic cell maturation and IL-10 production, thus suggesting a possible use as targeted imaging or antigen delivery tool [77].

\section{Concluding remarks and future perspectives}

Almost ten years have passed since the seminal review [2] suggesting the feasibility of antiadhesive therapy of infectious diseases and the potential of polyvalent carbohydrates in this context. The body of research has significantly increased and many potent antiadhesive molecules and materials have been proposed. Here we have highlighted two well-researched examples where a bacterial adhesin (FimH) or a human Pathogen Recognition Receptor (DC-SIGN) have been targeted with the aim of disrupting their interaction with host or viral glycans, respectively. A handful of other examples have been reported in the literature and recently reviewed, most notably for the control of $P$. aeruginosa adhesion and biofilm formation [78, 79]. Although FimHmediated adhesion seems to be effectively controlled also by monomannosides of extended aromatic aglycones, most of the current antiadhesive technologies employ high-valency glycoclusters. The platforms involved range from micelles to dendrimers, polymers and nanoparticles (gold nanoparticles, nanodiamonds), which has created the very active field of glyconanotechnology. Glycomimetic ligands used in this context can improve both the stability and the activity of the constructs. The field seems to be ripe for translation of these results into clinical 
studies, particularly on the FimH target. Sugar-protein interactions control bacterial adhesion also

for a number of other pathogens that have not been targeted for antiadhesion therapy, yet. The biochemical and structural data that have been accumulating on sugar-protein interactions, and the current ability to decipher the sequence and function of cell-associated glycans, will surely offer in the near future the possibility of developing novel tools to interfere in the infection process using materials that mimic the natural presentation of adhesion oligosaccharides.

\section{References}

1. Sharon, N. and Lis, H. (1989) Lectins as cell recognition molecules. Science 246, 227-234.

2. Sharon, N. (2006) Carbohydrates as future anti-adhesion drugs for infectious diseases. Biochim Biophys Acta 1760, 527-537.

3. Imberty, A., et al. (2008) Glycomimetics and glycodendrimers as high affinity microbial antiadhesins. Chem. Eur. J. 14, 7490-7499.

4. Bernardi, A., et al. (2013) Multivalent glycoconjugates as anti-pathogenic agents. Chem. Soc. Rev. 42, 4709-4727.

5. Pera, N.P. and Pieters, R.J. (2014) Towards bacterial adhesion-based therapeutics and detection methods. MedChem Comm 5, 1027-1035.

6. Cecioni, S., et al. (2015) Glycomimetics versus multivalent glycoconjugates for the design of high affinity lectin ligands. Chem Rev 115, 525-561.

7. Firon, N., et al. (1987) Aromatic alpha-glycosides of mannose are powerful inhibitors of the adherence of type 1 fimbriated Escherichia coli to yeast and intestinal epithelial cells. Infect. Immun. 55, 472-476.

8. Bouckaert, J., et al. (2005) Receptor binding studies disclose a novel class of high-affinity inhibitors of the Escherichia coli FimH adhesin. Mol Microbiol 55, 441-455.

9. Wellens, A., et al. (2008) Intervening with urinary tract infections using anti-adhesives based on the crystal structure of the FimH-oligomannose-3 complex. PLOS ONE 3, e2040.

10. Vanwetswinkel, S., et al. (2014) Study of the structural and dynamic effects in the FimH adhesin upon alpha-d-heptyl mannose binding. J Med Chem 57, 1416-1427.

11. Scharenberg, M., et al. (2012) Target Selectivity of FimH Antagonists. J. Med. Chem. 55, 9810-9816.

12. Fiege, B., et al. (2015) The Tyrosine Gate of the Bacterial Lectin FimH: A Conformational Analysis by NMR Spectroscopy and X-ray Crystallography. ChemBioChem 16, 1235-1246.

13. Sperling, O., et al. (2006) Evaluation of the carbohydrate recognition domain of the bacterial adhesin FimH: design, synthesis and binding properties of mannoside ligands. Org. Biomol. Chem. 4, 3913-3922.

14. Han, Z., et al. (2010) Structure-based drug design and optimization of mannoside bacterial FimH antagonists. J Med Chem 53, 4779-4792.

15. Han, Z., et al. (2012) Lead optimization studies on FimH antagonists: discovery of potent and orally bioavailable ortho-substituted biphenyl mannosides. J Med Chem 55, 3945-3959.

16. Klein, T., et al. (2010) FimH antagonists for the oral treatment of urinary tract infections: from design and synthesis to in vitro and in vivo evaluation. J Med Chem 53, 8627-8641. 
17. Jiang, X., et al. (2012) Antiadhesion therapy for urinary tract infections--a balanced PK/PD profile proved to be key for success. J Med Chem 55, 4700-4713.

18. Kleeb, S., et al. (2015) FimH antagonists: bioisosteres to improve the in vitro and in vivo PK/PD profile. J Med Chem 58, 2221-2239.

19. Gouin, S.G., et al. (2009) Synthetic multimeric heptyl mannosides as potent antiadhesives of uropathogenic Escherichia coli. ChemMedChem 4, 749-755.

20. Almant, M., et al. (2011) Clustering of Escherichia coli type-1 fimbrial adhesins by using multimeric heptyl alpha-D-mannoside probes with a carbohydrate core. Chem. Eur. J. 17, 10029-10038.

21. Bouckaert, J., et al. (2013) Heptyl $\alpha$-D-Mannosides Grafted on a $\beta$-Cyclodextrin Core To Interfere with Escherichia coli Adhesion: An In Vivo Multivalent Effect. Chem. Eur. J. 19, 7847-7855.

22. Lindhorst, T.K. and Dubber, M. (2015) Octopus glycosides: multivalent molecular platforms for testing carbohydrate recognition and bacterial adhesion. Carbohydr Res 403, 90-97 and references therein.

23. Durka, M., et al. (2011) The functional valency of dodecamannosylated fullerenes with Escherichia coli FimH--towards novel bacterial antiadhesives. Chem. Commun. 47, 13211323.

24. Lindhorst, T., et al. (1998) Inhibition of the type 1 fimbriae-mediated adhesion of Escherichia coli to erythrocytes by multiantennary $\alpha$-mannosyl clusters: The effect of multivalency. Glycoconj J 15, 605-613.

25. Yan, X., et al. (2015) Glycopolymers as Antiadhesives of E. coli Strains Inducing Inflammatory Bowel Diseases. Biomacromolecules 16, 1827-1836.

26. Lin, C.C., et al. (2002) Selective binding of mannose-encapsulated gold nanoparticles to type 1 pili in Escherichia coli. J Am Chem Soc 124, 3508-3509.

27. Hartmann, M., et al. (2012) Saccharide-modified nanodiamond conjugates for the efficient detection and removal of pathogenic bacteria. Chem. Eur. J. 18, 6485-6492.

28. Barras, A., et al. (2013) Glycan-functionalized diamond nanoparticles as potent E. coli antiadhesives. Nanoscale 5, 2307-2316.

29. Khanal, M., et al. (2015) Inhibition of type 1 fimbriae-mediated Escherichia coli adhesion and biofilm formation by trimeric cluster thiomannosides conjugated to diamond nanoparticles. Nanoscale 7, 2325-2335.

30. Knight, S. and Bouckaert, J. (2009) Structure, Function, and Assembly of Type 1 Fimbriae. In Glycoscience and Microbial Adhesion (Lindhorst, T.K. and Oscarson, S., eds), pp. 67-107, Springer Berlin Heidelberg.

31. Arnault, J.C. (2015) Surface Modifications of Nanodiamonds and Current Issues for Their Biomedical Applications. In Novel Aspects of Diamond (Yang, N., ed), pp. 85-122, Springer International Publishing.

32. Brument, S., et al. (2013) Thiazolylaminomannosides as potent antiadhesives of type 1 piliated Escherichia coli isolated from Crohn's disease patients. J Med Chem 56, 5395-5406.

33. Jarre, G., et al. (2011) Playing the surface game-Diels-Alder reactions on diamond nanoparticles. Chem Commun 47, 544-546.

34. Dondoni, A. and Marra, A. (2012) Recent applications of thiol-ene coupling as a click process for glycoconjugation. Chem. Soc. Rev. 41, 573-586.

35. Meinhardt, T., et al. (2011) Pushing the Functionality of Diamond Nanoparticles to New Horizons: Orthogonally Functionalized Nanodiamond Using Click Chemistry. Adv. Funct. Mater. 21, 494-500. 
36. Geijtenbeek, T.B.H., et al. (2000) DC-SIGN, a Dendritic Cell Specific HIV-1-Binding Protein that Enhances trans-Infection of T Cells. Cell 100, 587-597.

37. Geijtenbeek, T.B.H., et al. (2000) Identification of DC-SIGN, a Novel Dendritic Cell Specific ICAM-3 Receptor that Supports Primary Immune Responses. Cell 100, 575-585.

38. Mitchell, D.A., et al. (2001) A novel mechanism of carbohydrate recognition by the C-type Lectins DC-SIGN and DC-SIGNR: subunit organization and binding to multivalent ligands. J. Biol. Chem. 276, 28939-28945.

39. van Kooyk, Y. and Geijtenbeek, T.B. (2003) DC-SIGN: escape mechanism for pathogens. Nat Rev Immunol 3, 697-709.

40. Feinberg, H., et al. (2001) Structural basis for selective recognition of oligosaccharides by DC-SIGN and DC-SIGNR. Science 294, 2163-2166.

41. Guo, Y., et al. (2004) Structural basis for distinct ligand-binding and targeting properties of the receptors DC-SIGN and DC-SIGNR. Nat Struct Mol Biol 11, 591-598.

42. Feinberg, H., et al. (2007) Multiple modes of binding enhance the affinity of DC-SIGN for high mannose N-linked glycans found on viral glycoproteins. J. Biol. Chem. 282, 4202-4209.

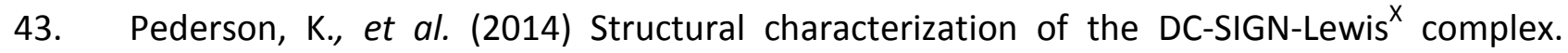
Biochemistry 53, 5700-5709.

44. Anderluh, M., et al. (2012) DC-SIGN Antagonists, a Potential New Class of Anti-Infectives. Curr. Med. Chem. 19, 992-1007.

45. Reina, J.J., et al. (2010) HIV microbicides: state-of-the-art and new perspectives on the development of entry inhibitors. Future Med. Chem. 2, 1141-1159.

46. Lepenies, B., et al. (2013) Targeting C-type lectin receptors with multivalent carbohydrate ligands. Adv.Drug Delver. Rev. 65, 1271-1281.

47. Prost, L.R., et al. (2012) Noncarbohydrate Glycomimetics and Glycoprotein Surrogates as DC-SIGN Antagonists and Agonists. ACS Chem. Biol. 7, 1603-1608.

48. Xiao, L., et al. (2013) Antibody-Mimetic Ligand Selected by mRNA Display Targets DC-SIGN for Dendritic Cell-Directed Antigen Delivery. ACS Chem. Biol. 8, 967-977.

49. Zhang, Q., et al. (2013) Sequence-Controlled Multi-Block Glycopolymers to Inhibit DC-SIGNgp120 Binding. Angew. Chem. Int. Ed. Engl. 52, 4435-4439.

50. Cipolla, L. ed (2015) Carbohydrate Chemistry: State of the Art and Challenges for Drug Development. Imperial College Press.

51. Lederman, M.M., et al. (2008) Topical application of entry inhibitors as "virustats" to prevent sexual transmission of HIV infection. Retrovirology 5, 116.

52. Garcia-Vallejo, J.J., et al. (2013) Glycodendrimers prevent HIV transmission via DC-SIGN on dendritic cells. Int. Immunol. 25, 221-233.

53. Gringhuis, S.I., et al. (2009) Carbohydrate-specific signaling through the DC-SIGN signalosome tailors immunity to Mycobacterium tuberculosis, HIV-1 and Helicobacter pylori. Nat. Immunol. 10, 1081-1088.

54. Garcia-Vallejo, J.J. and van Kooyk, Y. (2015) DC-SIGN: The Strange Case of Dr. Jekyll and Mr. Hyde. Immunity 42, 983-985.

55. Mangold, S.L., et al. (2012) Quinoxalinoneinhibitors of the lectin DC-SIGN. Chem. Sci. 3, 772-777 and references therein.

56. Lasala, F., et al. (2003) Mannosyl Glycodendritic Structure Inhibits DC-SIGN-Mediated Ebola Virus Infection in cis and in trans. Antimicrob. Agents Chemother. 47, 3970-3972.

57. Tabarani, G., et al. (2006) Mannose hyperbranched dendritic polymers interact with clustered organization of DC-SIGN and inhibit gp120 binding. FEBS Lett. 580, 2402-2408.

58. Luczkowiak, J., et al. (2013) Glycofullerenes inhibit viral infection. Biomacromolecules 14, 431-437. 
59. Ribeiro-Viana, R., et al. (2012) Virus-like glycodendrinanoparticles displaying quasiequivalent nested polyvalency upon glycoprotein platforms potently block viral infection. Nat. Commun. 3, 1303.

60. Kozlovska, T.M., et al. (1993) Recombinant rna phage $Q \beta$ capsid particles synthesized and self-assembled in Escherichia coli. Gene 137, 133-137.

61. Chalker, J.M., et al. (2011) A "Tag-and-Modify" Approach to Site-Selective Protein Modification. Acc. Chem. Res. 44, 730-741.

62. Becer, C.R., et al. (2010) High-Affinity Glycopolymer Binding to Human DC-SIGN and Disruption of DC-SIGN Interactions with HIV Envelope Glycoprotein. J. Am. Chem. Soc. 132, 15130-15132.

63. Schaeffer, E., et al. (2013) Dynamic micelles of mannoside glycolipids are more efficient than polymers for inhibiting HIV-1 trans-infection. Bioconjugate Chem 24, 1813-1823.

64. Wang, S.K., et al. (2008) Targeting the carbohydrates on HIV-1: Interaction of oligomannose dendrons with human monoclonal antibody $2 \mathrm{G} 12$ and DC-SIGN. Proc. Natl. Acad. Sci. U S A 105, 3690-3695.

65. Martinez-Avila, O., et al. (2009) Multivalent manno-glyconanoparticles inhibit DC-SIGNmediated HIV-1 trans-infection of human T cells. ChemBioChem 10, 1806-1809.

66. Garber, K.C.A., et al. (2010) A general glycomimetic strategy yields non-carbohydrate inhibitors of DC-SIGN. Chem. Commun. 46, 6747-6749.

67. Reina, J.J., et al. (2007) 1,2-Mannobioside mimic: synthesis, DC-SIGN interaction by NMR and docking, and antiviral activity. ChemMedChem 2, 1030-1036.

68. Thepaut, M., et al. (2013) Structure of a glycomimetic ligand in the carbohydrate recognition domain of C-type lectin DC-SIGN. Structural requirements for selectivity and ligand design. J Am Chem Soc 135, 2518-2529.

69. Sutkeviciute, I., et al. (2014) Unique DC-SIGN clustering activity of a small glycomimetic: A lesson for ligand design. ACS Chem Biol 9, 1377-1385.

70. Sattin, S., et al. (2010) Inhibition of DC-SIGN-mediated HIV infection by a linear trimannoside mimic in a tetravalent presentation. ACS Chem. Biol. 5, $301-312$.

71. Berzi, A., et al. (2012) A glycomimetic compound inhibits DC-SIGN-mediated HIV infection in cellular and cervical explant models. Aids 26, 127-137.

72. Varga, N., et al. (2013) Selective targeting of dendritic cell-specific intercellular adhesion molecule-3-grabbing nonintegrin (DC-SIGN) with mannose-based glycomimetics: synthesis and interaction studies of bis(benzylamide) derivatives of a pseudomannobioside. Chem. Eur. J. 19, 4786-4797.

73. Varga, N., et al. (2014) A multivalent inhibitor of the DC-SIGN dependent uptake of HIV-1 and Dengue virus. Biomaterials 35, 4175-4184.

74. Berzi, A. et al. (2014) Pseudo-Mannosylated DC-SIGN Ligands as Potential Adjuvants for HIV Vaccines. Viruses 6, 391-403

75. Ordanini, S., et al. (2015) Designing nanomolar antagonists of DC-SIGN-mediated HIV infection: ligand presentation using molecular rods. Chem. Commun. 51, 3816-3819.

76. Andreini, M., et al. (2011) Second generation of fucose-based DC-SIGN ligands : affinity improvement and specificity versus Langerin. Org Biomol Chem 9, 5778-5786.

77. Arosio, D., et al. (2014) Effective targeting of DC-SIGN by alpha-fucosylamide functionalized gold nanoparticles. Bioconjugate Chem. 25, 2244-2251.

78. Reymond, J.L., et al. (2013) Glycopeptide dendrimers as Pseudomonas aeruginosa biofilm inhibitors. Chem. Soc. Rev. 42, 4814-4822.

79. Boukerb, A.M., et al. (2014) Antiadhesive properties of glycoclusters against Pseudomonas aeruginosa lung infection. J Med Chem 57, 10275-10289. 
80. Qi, Z., et al. (2015) Multivalency at interfases: supramolecular carbohydrate-functionalized graphene derivatives for bacterial capture, release, and disinfection Nano Lett. 15, 60516057. 


\section{Glossary Box}

Adhesin: Components of bacterial surface that facilitate adhesion to other cells or surfaces. Adhesins are generally proteins in Gram-negative bacteria and polysaccharides in Gram-positive ones.

AIEC: Adherent Invasive E. coli, bacteria found in the small intestine of patients with Crohn's disease

CD209: Alternative name of DC-SIGN

C-lectins: or C-type lectins, are $\mathrm{Ca}^{++}$-dependent glycan-binding proteins that share structural homology in their Carbohydrate Recognition Domain.

CRD: Carbohydrate Recognition Domain, in a lectin, contains the sugar binding site

DC-SIGN: Dendritic Cell-Specific ICAM-3 Grabbing Non-integrin, also called CD209. It is a PRR membrane receptor of dendritic cells with specificity for mannosylated and fucosylated oligosaccharides.

DLS: Dynamic Light Scattering, is a photophysical technique that allows to determine the hydrodynamic radius of a particle

AFM: Atomic Force Microscopy, is a scanning probe microscopy with very high resolution (fractions of nanometers), that can measure local properties of sample surfaces

ND: NanoDiamonds, carbon-based nanoparticles obtained by detonation that are available in large quantities at reasonable price.

PRR: Pattern Recognizing Receptors, protein receptors expressed by cells of the innate immune system. They recognize invading pathogens binding to so-called Pathogen Associated Molecular Patterns that are often glycans displayed at the pathogen surface.

GND: Glyco NanoDiamonds, are nanodiamonds modified on their surface with carbohydrates or glycomimetics

gp120: Glycoprotein of the HIV envelope. It is recognized by DC-SIGN through interaction with its high-mannose glycans

FimH: Bacterial adhesin located on the tip of bacterial fimbriae and responsible for mannosemediated adhesion

ICAM3: A member of the intercellular adhesion molecule (ICAM) family Langerin: transmembrane C-type lectin, specifically expressed on Langheran cells. It is also called CD207 
$Q \beta$ : recombinant capsid protein derived from the bacteriophage $Q \beta$, it self-assembles to give icosahedral virus-like particles

UPEC: Uropathogenic $E$. coli, strains of $E$. coli that are the most common cause of urinary tract infections 


\section{Figure Legends}

Figure 1. Monovalent and polyvalent FimH antagonists. a) Monovalent FimH ligands butyl $\alpha$-Dmannoside 1 [8], heptyl $\alpha$-D-mannoside ( $\alpha-\mathrm{D}-\mathrm{HM}) \mathbf{2}$ [9], 3 [13], 4 [15] and 5 [17] for antiadhesion therapy of urinary tract infections; FimH antagonist $6[32,33]$ was developed for antiadhesion of AIEC to intestinal cells; b) Surface functionalization of small NDs 5 by Diels-Alder reaction of an in situ generated $o$-quinodimethide [27]. The sugar 10 is grafted using a thiol-ene reaction [34]; c) NDs surface functionalization as in [35]. The trivalent mannoside cluster $\mathbf{1 3}$ is grafted by amide bond formation [27]; d) and e) Functionalization of NDs 15 by esterification of surface hydroxyl groups to yield an azide or alkyne coating (in 16 and 19, respectively). The sugar (either mannose 17 or the trivalent cluster of $\alpha$-thiomannosides 20 ) is grafted by click reaction to the modified surface $[28,29]$; f) $\mathrm{N}$-( $\alpha$-mannopyranosyloxy)heptyl]methacrylamide (HMM) co-polymers 22 are powerful agglutinators of piliated E. coli. [25].

Figure 2. Naturally occurring oligosaccharides that are active DC-SIGN ligands. Mannose oligosaccharides $\mathrm{Man}_{9}$ and $\mathrm{Man}_{4}$ and the fucosylated trisaccharide Lewis ${ }^{\mathrm{X}}$.

Figure 3. High valency mannosides are powerful antagonists of viral recognition by DC-SIGN. a) Hypermannosylated glycodendriprotein [59]. Using nested levels of multivalency this construct has the size and shape of a viral capsid and carries up to 1620 mannose units b) Dynamic micelles of manno-glycolipids (27) inhibit HIV-1 trans-infection mediated by DC-SIGN expressing human dendritic cells [63].

Figure 4. Multivalent presentation of glycomimetics targeted against DC-SIGN. A modified shikimic acid 28 stands for mannose in glycopolymer 29, which antagonizes DC-SIGN binding to a highly mannosylated surface [66].

Figure 5. Mono- and multivalent mannose-based DC-SIGN ligands. Monovalent pseudomannobioside $\mathbf{3 0}$ and bis-benzylamido derivative 33. Pseudomannotrioside $\mathbf{3 1}$ and its tetravalent presentation on a Boltorn type dendron 32. Dendrimers 34-37: representative structures of dendrimers with valencies ranging from 3 to 18 prepared bearing either mannose or 
glycomimetics $\mathbf{3 0}$ or $\mathbf{3 3}$. The elongated hexavalent dendrimer 37, carrying 6 copies of $\mathbf{3 3}$ is a nanomolar inhibitor of HIV trans infection.

Figure 6. Fucose-based glycomimetic gold nanoparticles targeting DC-SIGN. An $\alpha$-fucosyl- $\beta$-alanyl amide mimics the Lewis ${ }^{\mathrm{X}}$ oligosaccharide in the coating of gold nanoparticles $\mathbf{3 8}$, that are potent DC-SIGN targeting tools [77]. 


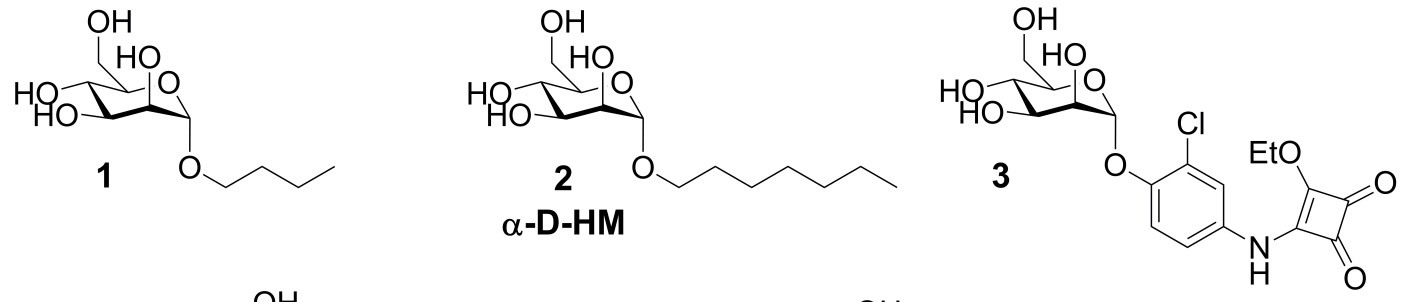<smiles>CC(=O)c1cccc(-c2ccc(OC3C(=O)C(O)C(O)C(O)C3O)c(C)c2)c1</smiles><smiles>O=[N+]([O-])c1ccc2c(c1)CCN2c1ccc(OC2C(O)C(O)C(O)C(O)C2O)cc1</smiles><smiles>Cc1nc(-c2cnccn2)sc1NC1C(O)C(O)C(O)C1O</smiles>

b)

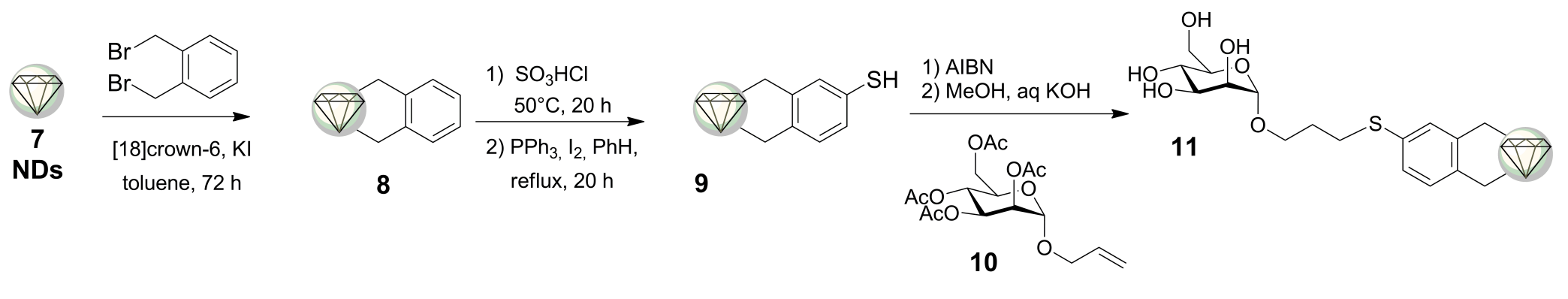

c)

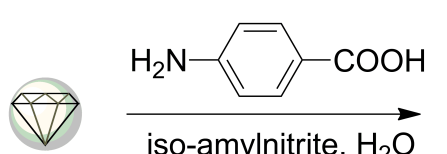

7

$80^{\circ} \mathrm{C}, 16 \mathrm{~h}$

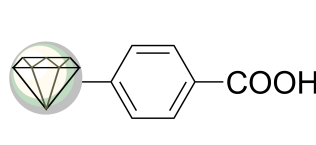

12

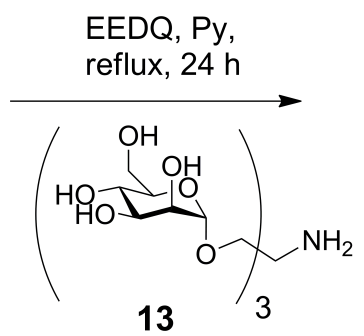

13

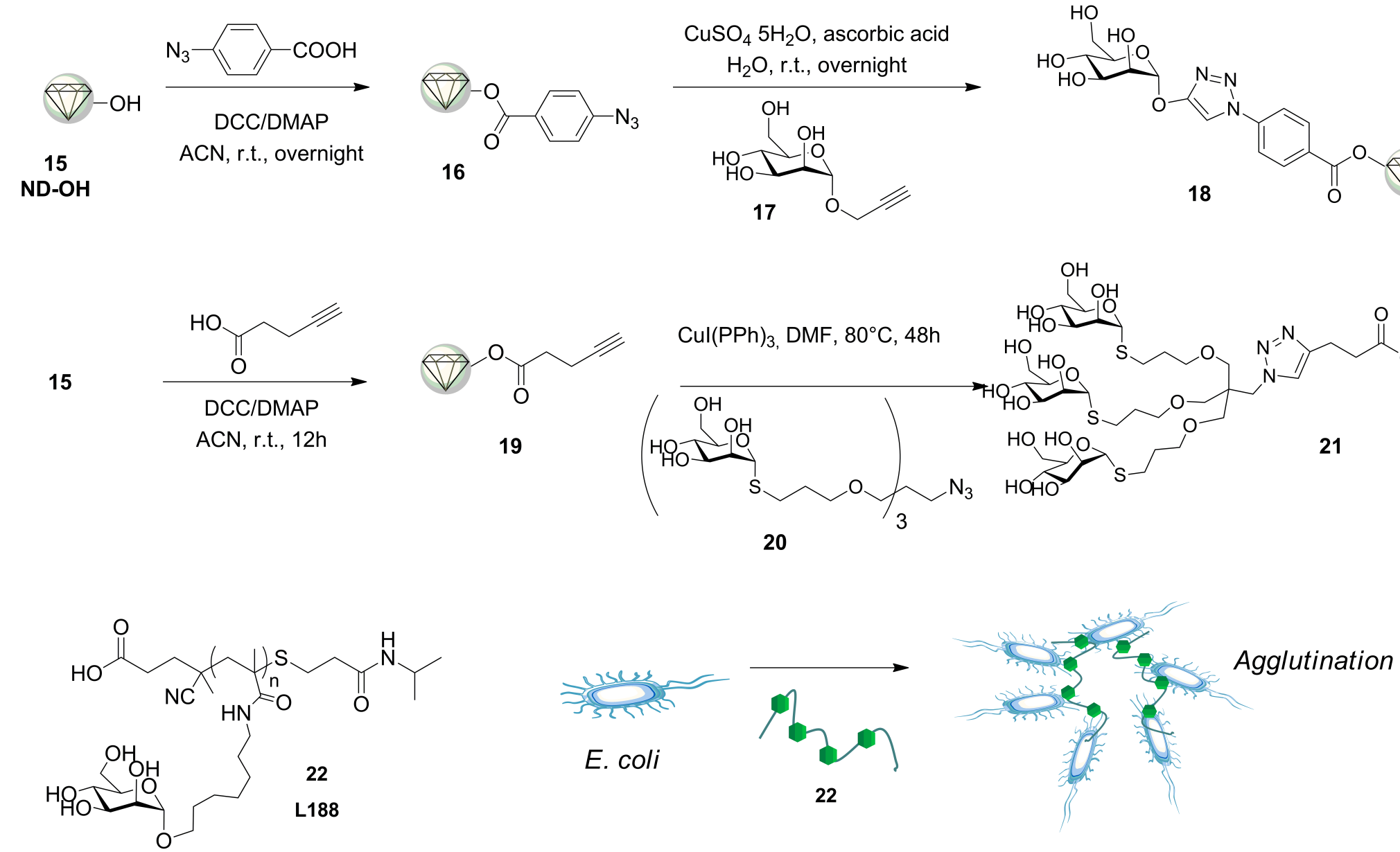

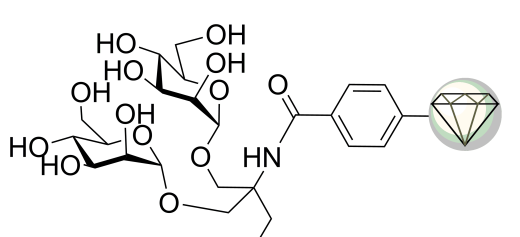

$$
14
$$

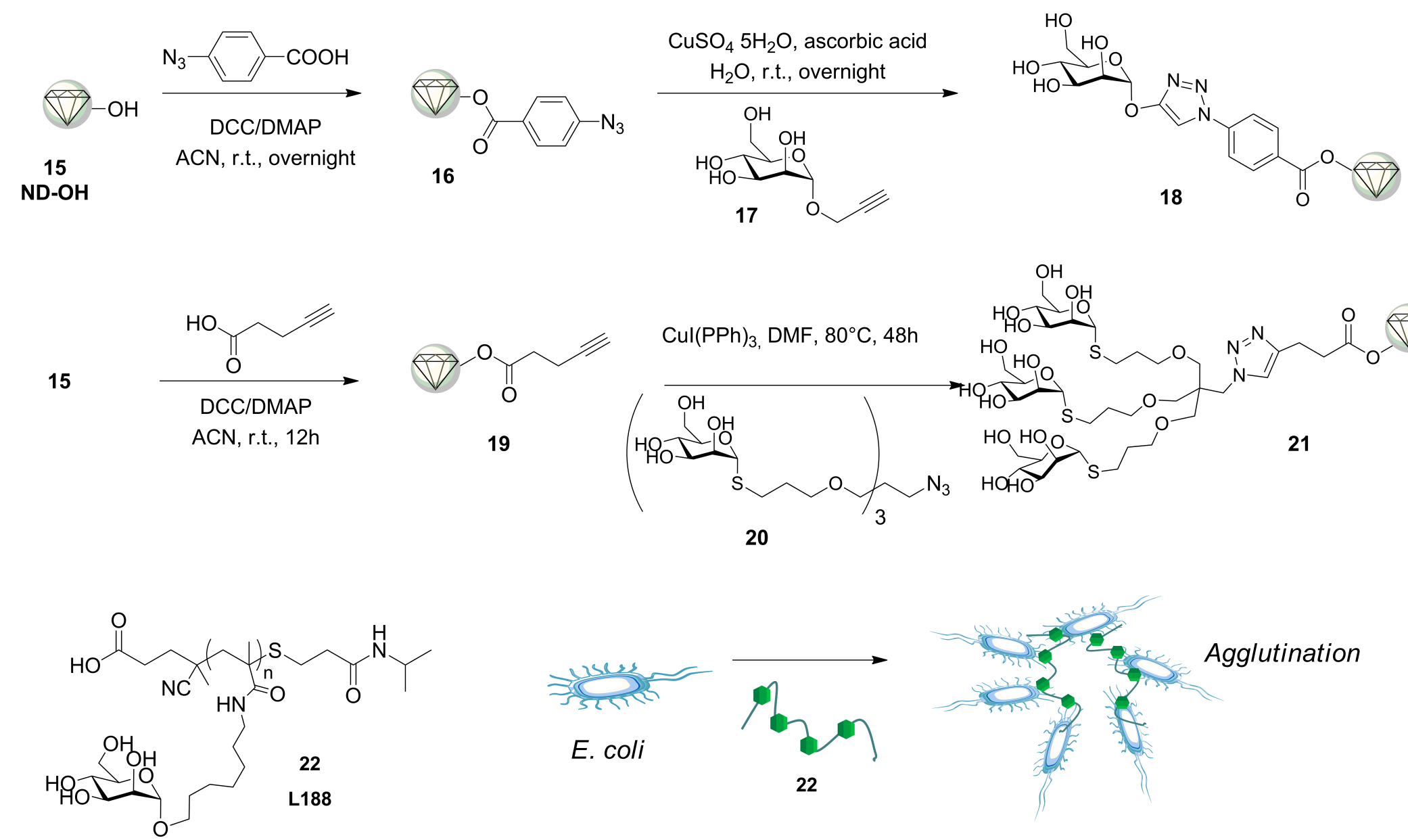

e) 

a)

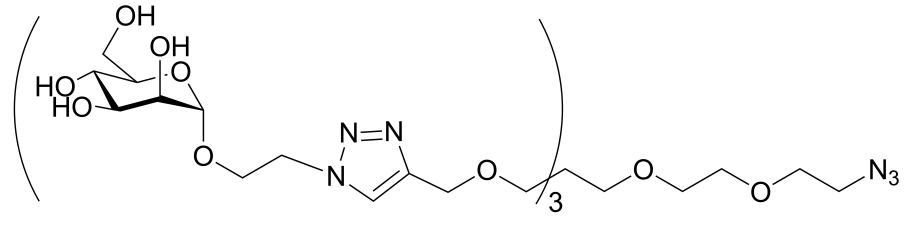

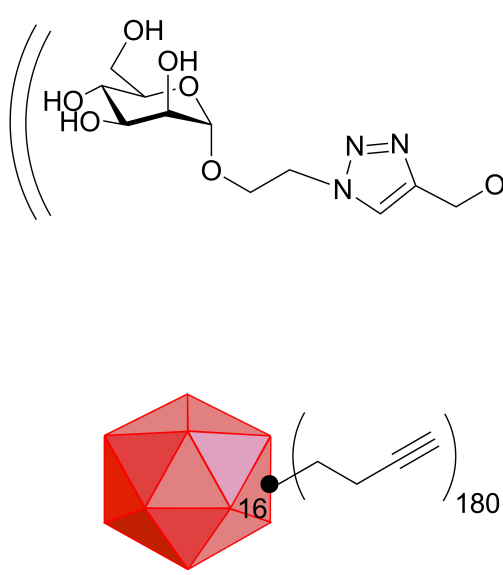

Qß-Hpg16 180

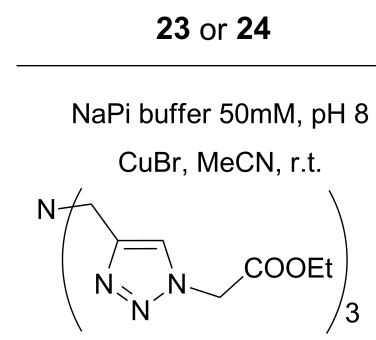

$\mathrm{CuBr}, \mathrm{MeCN}$, r.t.

b)

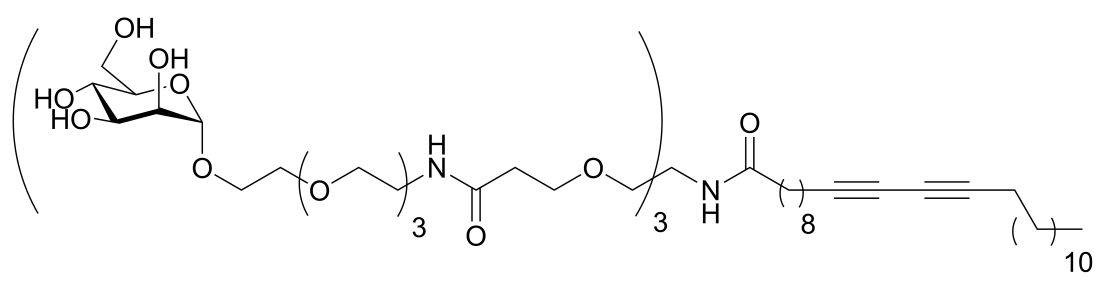

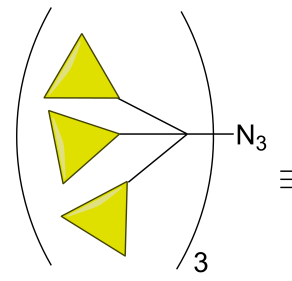

Ebola model infection test $25 \mathrm{n}=1 \quad \mathrm{IC}_{50} 9.62 \mathrm{nM}$ $26 \mathrm{n}=3 \quad I_{50} 910 \mathrm{pM}$

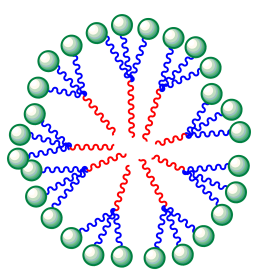

27 $N_{N=N}^{N} \overbrace{3} t_{0} \sim_{4}^{N_{3}}$ 
$\mathrm{OH}$

$-\mathrm{O} \mathrm{OH}$

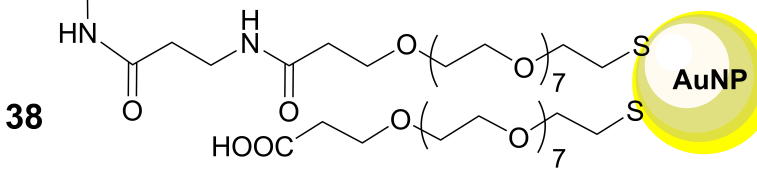

